\title{
The Screening and Treatment of Vitamin B12 Deficiency
}

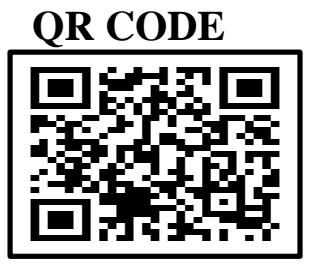

\section{PARUL SAHNI}

Vitamin B12 deficiency is now more prevalent than ever. It is a common cause of megaloblastic anemia, neuropsychiatric disorders, and other clinical manifestations. Screening is required in patients with single or multiple risk factors. Measurement of serum methylmalonic acid can be used to confirm deficiency in asymptomatic high-risk patients with low-normal levels of vitamin B12. Oral administration of high-dose vitamin B12 as well as intramuscular administration are effective as corrective therapy. This review briefs about the pharmacokinetics and pharmacodynamics of Vitamin B12, risk factors associated with its deficiency, screening methods and treatment options.

KEYWORDS: Vitamin B12, Cyanocobalamin, Cobalamin

\section{INTRODUCTION}

Vitamin B12 is a common name for a group of cobaltcontaining biologically active substances that differ in the structure of the ligand coupled to cobalt atom and called cobalamins. 'The main cobalamins in humans are hydroxocobalamin, adenosyl cobalamin and methylcobalamin, of which the latter two are active forms of coenzyme. ${ }^{2}$ In vitamin B12 preparations, cyanocobalamin is most commonly used as the active substance, which differs from other cobalamins in greater stability in the external environment and higher bioavailability, and therefore, it is cyanocobalamin that is usually referred to as "vitamin B12" in clinical practice. ${ }^{3}$ Vitamin $\mathrm{B} 12$ is involved in numerous physiologic processes required for normal growth, development, and metabolism of humans, and its deficiency can lead to severe, including life-threatening, disorders of various organs and systems. Studies conducted in recent decades worldwide have shown an increase in the prevalence of vitamin B12 deficiency, including its subclinical form, which may be due to both improved diagnosis and ageing of population and associated polymorbidity. This is the reason for the ongoing search for new approaches to the diagnosis and treatment of B12-deficient states.

Vitamin B12 enters the body with proteins of food from which it is released at low $\mathrm{pH}$ in the stomach lumen. ${ }^{4}$ After release, it binds to haptocorrin and remains complexed with it until proteolytic cleavage in the duodenum, where it binds to the Castle's intrinsic factor. After entering the brush border of the enterocyte, vitamin B12 separates from the Castle's intrinsic factor and enters the bloodstream, where it binds to transcobalamin II or haptocorrin, which deliver cobalamin to peripheral tissues and the liver. ${ }^{4}$ Most of the cobalamin transported by haptocorrin is metabolically inactive. Castle's intrinsic factor is responsible for absorption of approximately $99 \%$ of vitamin B12, while $0.5-5 \%$ is absorbed from the mucosa by simple diffusion all along the gastrointestinal tract. ${ }^{4-}$ 5 Vitamin B12 is distributed to almost all organs and tissues. Its steady-state concentrations are produced most rapidly in plasma, red blood cells, liver and kidneys, more slowly in adipose tissue, skeletal muscles, bone and brain. ${ }^{6}$ The main site of vitamin B12 deposition is the liver, where up to $90 \%$ of its reserves are concentrated. ${ }^{6}$ Up to $3-7 \mu$ g of vitamin B12 are excreted with bile from liver into the intestines per day, and about $75 \%$ of this amount is reabsorbed. Enterohepatic circulation determines the long half-life of vitamin B12 from the liver, about 12 months. ${ }^{7}$ In the body, cyanocobalamin is converted to coenzyme forms, methylcobalamin and 5-deoxyadenosylcobalamin. Methylcobalamin is involved in numerous transmethylation reactions and acts as a coenzyme for methionine synthase catalyzing the conversion of homocysteine to methionine required for the formation of S-adenosyl methionine, a universal methyl group donor for almost 100 different substrates, including DNA, RNA, hormones, proteins, and lipids.,4 Methionine is required for the conversion of folic acid to folinic acid, which provides a normoblastic type of hematopoiesis. 5-deoxyadenosylcobalamin is a cofactor for the reaction of L-methylmalonyl-CoA isomerization 
in succinyl-CoA during the degradation of propionic acid, which plays an important role in the metabolism of carbohydrates and lipids. 5 Cobalamin deficiency results in accumulation of methylmalonic acid and neurologic symptoms. Succinyl-CoA is also necessary for the synthesis of hemoglobin. ${ }^{5}$ Cells that divide rapidly need vitamin B12 most. Vitamin B12 deficiency leads to impaired proliferation of hematopoietic cells and epithelial cells, increased concentrations of propionic and methylmalonic acids that have a damaging effect on the myelin sheaths of nerve fibers, and Hcy, which is a risk factor for cardiovascular complications. ${ }^{8}$ Clinically, vitamin B12 deficiency can manifest as lesions of various organs and systems. The classic manifestation of B12 hypovitaminosis is considered pernicious (B12-deficient) anemia associated with insufficient production of the Castle's intrinsic factor by parietal cells of the stomach.

However, in recent years, neurological manifestations of vitamin B12 deficiency have become a priority, such as sensory and motor disorders, ataxia, cognitive impairment, including those leading to dementia and mental disorders, which often occur in the absence of hematological disorders. 9 In epidemiological studies, vitamin B12 deficiency was associated with the occurrence of infertility, delayed development of children, as well as thrombosis, including thrombosis of cerebral venous sinuses. ${ }^{7,10}$ Thrombosis was observed in severe vitamin B12 deficiency, resulting in marked hyperhomocysteinemia. ${ }^{7}$ In the elderly, vitamin B12 deficiency was also associated with decreased tolerance to orthostatic hypotension, being an important cause of falls and associated fractures ${ }^{11}$, with an increased risk of age-related macular degeneration ${ }^{12}$, senile asthenia ${ }^{13}$, and sarcopenia ${ }^{14}$, which are important causes of disability. Vitamin B12 deficiency may develop due to its insufficient intake with food, increased need, malabsorption, disturbance in transport or metabolism. Since vitamin B12 metabolism in the body is complicated, it can cause vitamin deficiency at any stage. The latter is especially typical for the elderly due to age-related physiological changes, polymorbidity and polypharmacy. ${ }^{2}$ In economically developed countries, the most common causes of vitamin B12 deficiency include atrophic gastritis, administration of medicinal products that reduce gastric acidity, metformin, and chloramphenicol. ${ }^{15}$ The elderly are at highest risk of developing vitamin B12 deficiency, especially those over 75 years old. ${ }^{3}$ In economically developed countries, the prevalence of vitamin B12 deficiency is about $6 \%$ in people under 60 years of age, about $20 \%$ in those over 60 years of age $\mathrm{e}^{16}$, and $23-37 \%$ in those over 80 years of age $^{17}$, but its actual prevalence in the elderly may be even higher because symptoms of deficiency in the elderly may be masked by age-related changes and comorbidities. ${ }^{2}$ About $10-25 \%$ of the elderly have subclinical vitamin B12 deficiency characterized by low serum levels of the vitamin in the complete absence of clinical symptoms or with mild general symptoms that are often mistakenly associated with other disorders. ${ }^{18} \mathrm{It}$ should be noted that the development of clinical symptoms of vitamin B12 deficiency since the onset of etiological factor can be delayed by $3-10$ years due to its high body stores and long half life in the liver. ${ }^{12,18}$ However, even subclinical vitamin B12 deficiency can lead to increased serum Hcy concentration and an increased risk of cardiovascular diseases ${ }^{19,20}$ and, apparently, cognitive impairment. ${ }^{21}$ The main development mechanism of vitamin B12 deficiency in the elderly, occurring in $40-70 \%$ of cases, is the foodcobalamin malabsorption syndrome, a disorder characterized by the inability to release cobalamin from the link with food proteins, which leads to the unavailability of the vitamin to form the complex with the Castle's intrinsic factor. ${ }^{22,23}$ Factors contributing to this syndrome include atrophic gastritis, Helicobacter pylori infection, antisecretory drugs, alcohol abuse, gastric bypass or certain other GI surgeries, and chronic exocrine pancreatic insufficiency. ${ }^{22}$ It should be kept in mind that absorption of vitamin B12 from food worsens as early as at the age of over 50 years. ${ }^{3}$ In addition, elderly people with normal serum vitamin B12 values may have its functional deficiency due to oxidative stress. ${ }^{23}$ The risk of both pernicious anemia and neuropsychiatric disorders is significantly higher in the elderly than that in younger people.9,24 Many elderly patients, especially those with comorbidity, such as renal insufficiency and/or diabetes mellitus, even with normal serum levels of cobalamin and HoloTC, may have elevated MMA levels, which led to the hypothesis that they are resistant to cobalamin and, consequently, should be prescribed higher doses of vitamin B12.. 25 Other vulnerable population categories for vitamin B12 deficiency include vegans and strict vegetarians, pregnant and breastfeeding women, infants of the first 2 years of life, particularly those who were exclusively breastfed, those with pernicious anemia, celiac disease, and Crohn's disease, those who underwent GI surgery resulting in the impaired production of the Castle's intrinsic factor and hydrochloric acid secretion. ${ }^{10,25}$ The need for vitamin B12 is also increased in people who abuse alcohol, smokers, and those with HIV infection, tuberculosis, kidney disease, hyperthyroidism, and diabetes mellitus. ${ }^{10,25}$ Given the potentially serious consequences of vitamin B12 deficiency and the 
irreversibility of some of them, in particular neurological ones in case of delayed initiation of treatment ${ }^{26}$, it is necessary to identify the deficiency in early stages and to prescribe treatment in a timely manner. ${ }^{27}$ Therefore, screening for vitamin B12 deficiency in populations with risk factors is advisable. According to experts, screening for vitamin B12 deficiency may be warranted in patients with one or more risk factors, such as gastric or small intestine resection, inflammatory bowel disease, administration of metformin for more than 4 months, administration of proton pump inhibitors or $\mathrm{H} 2$ blockers for more than 12 months, veganism or strict vegetarianism, and age over 75 years. ${ }^{3}$ Since the risk of serious consequences of vitamin B12 deficiency, including pernicious anemia, cognitive and behavioral disorders, depression, myocardial infarction, stroke is highest in the elderly, many researchers advise screening for vitamin B12 deficiency in all the elderly. ${ }^{22}$ It is possible that in addition to the elimination of neurological and hematological symptoms, vitamin B12 may have other favorable effects in the elderly, such as antidepressant, antipsychotic ${ }^{22}$, analgesic, and opioid-sparing. ${ }^{28}$ As of now, there is no gold standard for diagnosing vitamin B12 deficiency. Furthermore, there is no commonly accepted determination of normal vitamin B12 levels. The World Health Organization considers the vitamin B12 status in adults to be sufficient at serum levels greater than $221 \mathrm{pmol} / \mathrm{L}$. Levels of 148 to $221 \mathrm{pmol} / \mathrm{L}$ are considered low, and less than $148 \mathrm{pmol} / \mathrm{L}$ are considered B12 deficiency. ${ }^{29}$ Several circulating biomarkers can be identified for diagnostic purposes: total B12, active B12 (HoloTC), and 2 metabolic markers, Hcy and MMA. The determination of each of these biomarkers has its limitations, as their level may be altered by some nonspecific factors, therefore taking into account the availability and cost of tests, a stratified stepwise approach, starting with the determination of total vitamin B12 level and complete blood count are typically advised to assess B12 deficiency. ${ }^{27}$

Patients with serum cobalamin levels IN the range of 110-148 pmol/L are recommended to be retested in 1-2 months. ${ }^{30}$ Patients with persistent suboptimal cobalamin levels should be tested for titers of Castle's intrinsic factor antibodies and given low doses of oral vitamin B12. Such patients should be warned of the need to closely monitor for potential neurologic symptoms and if they occur, seek medical attention immediately. Patients with positive tests for the Castle's intrinsic factor antibodies should be treated according to the therapeutic guidelines for pernicious anemia. In patients with negative test results, the cobalamin levels should be re-evaluated in 3-4 months and, if remained low, the biochemical testing should be continued to confirm biochemical deficiency. ${ }^{31}$ It is a paradox that some patients with symptoms of vitamin B12 deficiency have elevated serum cobalamin levels with parallel elevation of MMA and Hcy markers. This functional deficiency of cobalamin, which is more often asymptomatic, can develop with increased binding of vitamin B12 to haptocorrin rather than transcobalamin II, followed by a decrease in the vitamin delivery to peripheral cells. ${ }^{4}$ These patients are recommended to receive trial therapy with hydroxocobalamin..$^{30}$ Elevated B12 levels are usually associated with liver disease and hematologic abnormalities, but may occur in some cancers ${ }^{4}$, suggesting that some cancers may impair the metabolism of vitamin B12. Repeated intramuscular injections of vitamin B12 have been considered the gold standard of therapy for B12-deficient states, but they have a number of limitations that can degrade quality of life. First, injections are painful, causing fear of treatment or refusal in a number of patients. Second, they cause adverse reactions at the injection site, including intramuscular hematomas, especially in individuals receiving concomitant antithrombotic therapy. ${ }^{2}$

Injectable forms of vitamin B12 are more allergenic than tablets. In addition, injectable products require consumables and healthcare professionals for their administration, which in combination with an increased risk of adverse reactions and the high cost of injectable therapy, leads to an additional economic burden for patients themselves and the healthcare system. Given the fact that many patients, such as those with pernicious anemia or irreversible digestive tract disorders, require lifelong vitamin B12 replacement therapy, it can have significant negative medical and economic effects. An alternative to injectable products is the high-dose oral cyanocobalamin agents, which create sufficient cobalamin concentrations in the blood through diffuse absorption mechanism, including in patients with cobalamin malabsorption of various etiologies, including Castle's intrinsic factor deficiency, atrophic gastritis, surgeries and antisecretory drugs. The efficacy and safety of oral drugs containing 1,00o $\mu \mathrm{g}$ of cyanocobalamin in individuals with vitamin B12 deficiency have been demonstrated in numerous studies, including comparative ones with intramuscular vitamin B12, and in metanalyses. 


\section{CONCLUSION}

Research work conducted in recent years across the globe indicates an increase in the prevalence of vitamin B12 deficiency. Further studies are required to detail the features of the pharmacokinetics and pharmacodynamics of vitamin B12, the causes and risk factors for the development and clinical manifestations of its deficiency. Also, the concerned professionals should provide updated modern recommendations for screening and diagnosis of vitamin B12 deficiency.

\section{REFERENCES}

1. Kennedy DO. B vitamins and the brain: Mechanisms, dose and efficacy -A review. Nutrients. 2016;8:68. https://doi.org/10.3390/nu8020068.

2. Andrès E, Zulfiqar AA, Vogel T. State of the art review: oral and nasal vitamin B12 therapy in the elderly. QJM. 2020;113(1):5-15. https://doi.org/10.1093/qjmed/hczo46

3. Langan RC, Goodbred AJ. Vitamin B12 Deficiency: Recognition and Management. Am Fam Physician. 2017;96(6):384-9.

4. Shipton MJ, Thachil J. Vitamin B12 deficiency - A 21st century perspective. Clin Med (Lond). 2015;15(2):145-50. https://doi.org/10.7861/clinmedicine.15-2-14

5. Romain M, Sviri S, Linton DM, Stav I, van Heerden $\mathrm{PV}$. The role of Vitamin B12 in the critically ill - a review. Anaesth Intensive Care. 2016; 44(4):447-52.

6. Alpers DH. Absorption and blood/cellular transport of folate and cobalamin: Pharmacokinetic and physiological considerations. Biochimie. 2016; 126:52-6. https://doi.org/10.1016/j.biochi.2015.11

7. Stabler SP. Clinical practice. Vitamin B12 deficiency. $\mathrm{N}$ Engl J Med. 2013;368(2):14916o.https://doi.org/10.1056/NEJMcp1113996

8. Carmel R. Current concepts in cobalamin deficiency. Annu Rev Med. 2000; 51:357-375. https://doi.org/10.1146/annurev.med.51.1.357

9. Andrès E, Loukili NH, Noel E, Kaltenbach G, Abdelgheni MB, Perrin AE, Noblet-Dick M, Maloisel F, Schlienger JL, Blicklé JF. Vitamin B12 (cobalamin) deficiency in elderly patients. CMAJ. 2004;171(3):251-259. https://doi.org/10.1503/cmaj.1031155

10. Sukumar N, Saravanan P. Investigating vitamin B12 deficiency. $\quad$ BMJ. 2019; 365:11865. https://doi.org/10.1136/bmj.l1865

11. Moore A, Ryan J, Watts M, Pillay I, Clinch D, Lyons D. Orthostatic tolerance in older patients with vitamin B12 deficiency before and after vitamin B12 replacement. Clin Auton Res. 2004;14(2):67-71. https://doi.org/10.1007/s10286-004-0142-X

12. Gopinath B, Flood VM, Rochtchina E, Wang JJ, Mitchell P. Homocysteine, folate, vitamin B-12, and 10-y incidence of age-related macular degeneration. Am J Clin Nutr. 2013;98(1):129-135. https://doi.org/10.3945/ajcn.112.057091

13. Soh Y, Won CW. Association between frailty and vitamin $\mathrm{B} 12$ in the older Korean population. Medicine (Baltimore). 2020;99(43):e22327. https://doi.org/10.1097/MD.ooooooooooo22327 14. Ates Bulut E, Soysal P, Aydin AE, Dokuzlar O, Kocyigit SE, Isik AT. Vitamin B12 deficiency might be related to sarcopenia in older adults. Exp Gerontol. 2017;95:136-40.

https://doi.org/10.1016/j.exger.2017.05.017

15. Green R, Allen LH, Bjørke-Monsen AL, Brito A, Guéant JL, Miller JW, Molloy AM, Nexo E, Stabler S, Toh BH, Ueland PM, Yajnik C. Vitamin B12 deficiency. Nat Rev Dis Primers. 2017;3:17040. https://doi.org/10.1038/nrdp.2017.40

16. Hunt A, Harrington D, Robinson S. Vitamin B12 deficiency. BMJ. 2014; 349:95226. https://doi.org/10.1136/bmj.g5226

17. Johnson MA, Hausman DB, Davey A, Poon LW, Allen RH, Stabler SP; Georgia Centenarian Study. Vitamin B12 deficiency in African American and white octogenarians and centenarians in Georgia. J Nutr Health Aging. 2010;14(5):339-45. https://doi.org/10.1007/s12603-010oo77-y

18. Carmel R. How I treat cobalamin (vitamin B12) deficiency. Blood. 2008; 112(6):2214-21. https://doi.org/10.1182/blood-2008-03-040253

19. Ammouri W, Tazi ZM, Harmouche H, Maamar M, Adnaoui M. Venous thromboembolism and hyperhomocysteinemia as first manifestation of pernicious anemia: a case series. J Med Case Rep. 2017;11(1):25o. https://doi.org/10.1186/s13256-017-1415-z

20. Kim J, Kim $\mathrm{H}_{1}$, Roh $\mathrm{H}$, Kwon Y. Causes of hyperhomocysteinemia and its pathological significance. Arch Pharm Res. 2018;41(4):372-83. https://doi.org/10.1007/s12272-018-1016-4

21. Carmel R. Subclinical cobalamin deficiency. Curr Opin Gastroenterol. 2012; 28(2):151-8. https://doi.org/10.1097/MOG.obo13e3283505852

22. Wong CW. Vitamin B12 deficiency in the elderly: is it worth screening? Hong Kong Med J. 2015;21(2):155-164. https://doi.org/10.12809/hkmj144383

23. Solomon LR. Functional cobalamin (vitamin B12) deficiency: role of advanced age and disorders associated with increased oxidative stress. Eur J Clin Nutr. 2015;69:687-92. https://doi.org/10.1038/ejcn.2014.272

24. Zik C. Late Life Vitamin B12 Deficiency. Clin Geriatr Med. 2019;35(3):319-25. 
https://doi.org/10.1016/j.cger.2019.03.004 25. Solomon LR. Disorders of cobalamin (vitamin B12) metabolism: emerging concepts in pathophysiology, diagnosis and treatment. Blood Rev. 2007;21(3):113-30. https://doi.org/10.1016/j.blre.2006.05.001

26. Briani C, Dalla Torre C, Citton V, Manara R, Pompanin S, Binotto G, Adami F. Cobalamin deficiency: clinical picture and radiological findings. Nutrients. 2013;5(11):4521-39. https://doi.org/10.339o/nu5114521

27. Jarquin Campos A, Risch L, Nydegger U, Wiesner J, Vazquez Van Dyck M, Renz H, Stanga Z, Risch M. Diagnostic Accuracy of Holotranscobalamin, Vitamin B12, Methylmalonic Acid, and Homocysteine in Detecting B12 Deficiency in a Large, Mixed Patient Population. Dis Markers. 2020;2020: 7468506. https://doi.org/10.1155/2020/7468506

Source of support: Nil, Conflict of interest: None declared
28. Buesing S, Costa M, Schilling JM, Moeller-Bertram T. Vitamin B12 as a Treatment for Pain. Pain Physician. 2019;22(1):45-52.

29. Hannibal L, Lysne V, Bjørke-Monsen AL, Behringer S, Grünert SC, Spiekerkoetter U, Jacobsen DW, Blom HJ. Biomarkers and Algorithms for the Diagnosis of Vitamin B12 Deficiency. Front Mol Biosci. 2016;3:27. https://doi.org/10.3389/fmolb.2016.00027

30. Devalia V, Hamilton MS, Molloy AM; British Committee for Standards in Haematology. Guidelines for the diagnosis and treatment of cobalamin and folate disorders. $\mathrm{Br}$ J Haematol. 2014;166(4):496-513. https://doi.org/10.1111/bjh.12959

31. Arendt JFH, Sørensen HT, Horsfall LJ, Petersen I. Elevated Vitamin B12 Levels and Cancer Risk in UK Primary Care: A THIN Database Cohort Study. Cancer Epidemiol Biomarkers Prev. 2019;28(4):814-21. https://doi.org/10.1158/1055-9965.EPI-17-1136
Cite this article as:

Sahni P. The Screening and Treatment of Vitamin B12 Deficiency. Int Healthc Res J. 2021;5(4):RV1-RV5. https://doi.org/10.26440/IHRJ/0504.07439

\section{AUTHOR AFFILIATIONS: (*Corresponding Author)}

1. BDS, Pharmacovigilance Expert, Haryana, India 